\title{
P 11 PHENYTOIN DOSING AND LEVEL MONITORING
}

Amanda Bevan, ${ }^{1}$ Habib Wardak ${ }^{2}$. ${ }^{1}$ Southampton Children's Hospital; ${ }^{2}$ University Hospital Southampton NHS Foundation Trust

10.1136/archdischild-2015-308634.19

Introduction Phenytoin is a first-line anticonvulsant used for status epilepticus and neurosurgery prophylaxis. It is highly protein bound (about 90\%), primarily to albumin. It has a narrow therapeutic range and has non-linear pharmacokinetics. A guideline on safe prescribing, administration and monitoring of IV phenytoin on paediatric wards was written recently. 
Aim To audit the prescribing and therapeutic level monitoring of phenytoin against the guideline.

Method A retrospective audit of all children aged 0-12 years who had a phenytoin level measured in the laboratory between Sept 1 st 2012 and Aug 31st 2013. The children were identified from biochemistry records. Clinical information was obtained from the patient case notes; biochemistry reporting system (e-Quest), nursing notes and prescription charts were used to collect information in regards to timing of loading doses and samples. Data was analysed using Microsoft Excel.

Results Sixty-nine patients were identified, five were excluded due to age, eighteen other patients either had their loading doses given in other hospitals and no data was available or the notes could not be obtained. Phenytoin dosing was analysed in fifty-one patients.

$67 \%$ of patients were loaded correctly $(20 \mathrm{mg} / \mathrm{kg})$ according to the local guideline, $31 \%$ were given a lower dose than recommended and only $2 \%$ were overloaded.

In $39 \%$ of patients blood samples were taken within 2-4 hours after loading dose. In these patients the levels were within the therapeutic range in $55 \%$ of patients.

Another $58 \%$ of patients had levels measured but not at the correct time. The other $3 \%$ of patients did not have levels measured.

In $37 \%$ of patients, the maintenance dose was started 12 hours after loading dose but not in 39\% of patients; the remaining 24\% did not receive maintenance dosing with phenytoin.

Conclusion This audit has shown that there is room for improvement in the way patients receiving phenytoin are managed, particularly in relation to blood level monitoring and loading doses. There is also a problem with documentation of loading doses given in other hospitals prior to patients being retrieved to the regional tertiary centre. All prescribers should be made aware of the phenytoin guideline. Pharmacists have a vital role in the implementation and adherence to this guideline and in the education of other health care professionals. Staff should be encouraged to report incidents of delayed doses, over doses and when inappropriate blood samples have been taken. The introduction of an electronic prescribing system locally might allow blood level requests to be prescribed. 